\title{
Uma Revisão Sobre a Dominância Manual em Gêmeos
}

\author{
A Review of Handedness In Twins
}

Ana Flávia Lima Teles da Hora', William Lee Berdel Martin ${ }^{2}$

\section{RESUMO}

O método de gêmeos tem sido uma possibilidade de se investigar a gênese e o padrão de lateralizaçáo do sistema neuromotor, visto que, a comparação entre os gêmeos monozigóticos, considerados geneticamente idênticos com os gêmeos dizigóticos que compartilham apenas $50 \%$ da carga genética, viabiliza a análise da magnitude da influência genética e ambiental. Objetivo. Delinear por meio de uma revisão de dados de estudos com gêmeos as principais teorias que procuram descrever a organização motora subjacente a dominância manual tanto em destros quanto canhotos em gêmeos monozigóticos e dizigóticos. Método. Foi realizada uma extensa revisão de literatura focada na dominância manual em gêmeos por meio de consulta em banco de dados das seguintes fontes: COMUTE/BIREME, LILACS, Science Direct, and Cochrane Cogprints. Resultados. Foram selecionados artigos publicados entre os anos de 1970 a 2009. Conclusáo. Não há um consenso entre os cientistas sobre a divergência da dominância manual e a prevalência de assimetria do sistema neuromotor. Sem uma descrição compreensível e extensiva das expressóes fenotípicas derivadas dos grupos, é muito difícil avaliar certas hipóteses alternativas referentes às possíveis variações no padrão de especializações neuromotoras em gêmeos.

Unitermos. Gêmeos Monozigóticos, Gêmeos Dizigóticos, Lateralidade Funcional.

Citaçáo. Hora AFLT, Martin WLB. Uma Revisão Sobre a Dominância Manual em Gêmeos.

\section{ABSTRACT}

The twin method affords an opportunity to investigate the genesis and functional pattern of lateralization in the neuromotor system of both monozygotic and dizygotic twin pairs, which, respectively, share 100 and $50 \%$ of their genetic structure in common. The method also permits an analysis of the degree to which genetic and environmental factors interact in the development of lateral dominance. Objective. By means of a review of data from twin studies to delineate the principal theories seeking to describe the motor organization underlying handedness in both left- and right-handed monozygotic and dizygotic twins. Method. An extensive review was undertaken on handedness data from twin research conducted since 1970 by consulting the following database sources: COMUTE/BIREME, LILACS, Science Direct, and Cochrane Cogprints. Results. We selected articles published between 1970 and 2009. Conclusion. There was no clear consensus among researchers regarding the origin of lateral asymmetries in the neuromotor network, and the development of handedness in twins. Without a thorough and comprehensive description of phenotypic lateral attributes associated with handedness it was very difficult to evaluate the validity current alternate theories regarding variations in the pattern of neuromotor specialization in monozygotic and dizygotic twins.

Keywords. Monozygotic and Dizygotic Twins, Handedness, Neuromotor Lateralization.

Citation. Hora AFLT, Martin WLB. A Review of Handedness In Twins.
Trabalho realizado na Universidade Federal do Pará, UFPA, Belém-PA, Brasil.

1.Psicóloga, Mestre em Teoria e Pesquisa do Comportamento (NTPC) da Universidade Federal do Pará - NTPC/UFPA, Belém-PA, Brasil. Bolsista CNPQ.

2.Antropólogo, Doutor em Psicologia e Antropologia Biológica pela University of Illinois (EUA), Professor associado da Universidade Federal do Pará, orientador do NTPC, Belém-PA, Brasil.
Endereço para correspondência: William Lee Berdel Martin

Universidade Federal do Pará Centro de Filosofia e Ciências Humanas Departamento de Psicologia Experimental Av. Augusto Correa, s/n, Guamá Tel/Fax: (91) 32018477 / (91) 32017662 CEP 66065-115, Belém-PA, Brasil. E-mail: wlbm2011@gmail.com; afmundial@hotmail.com 


\section{INTRODUÇÃO}

Dominância manual abrange tarefas unimanuais, que exigem movimentos complexos, coordenação motora fina e/ou força muscular, que por sua vez são mediados pelo sistema neuromotor no hemisfério contralateral a mâo usada ${ }^{1}$. No caso da escrita, por exemplo, os resultados de estudos de destros normais, que não foram obrigados a escrever com a mão direita, usando técnicas de neuroimagem, verificaram um padrão de ativação neural no hemisfério esquerdo, principalmente nos lobos frontais e parietais. Em canhotos a ativação ocorreu nas mesmas áreas no hemisfério direito ${ }^{2-5}$.

Com base na análise de implementos líticos préhistóricos, há um consenso que, desde quando os precursores hominídeos surgiram (começando com Homo habilis), a espécie humana tem sido predominantemente destra na dominância manual e por inferência em outras assimetrias laterais. Estima-se que entre 1 a 30\% dos hominídeos eram canhotos, podendo esta porcentagem variar em decorrência do período e do local ${ }^{6-9}$.

Em muitos levantamentos, a percentagem de canhotos também muda em função de grupo étnico, gênero e em alguns grupos com características especiais. Por exemplo, foi amplamente comprovado que canhotismo ocorre mais frequentemente no sexo masculino que no feminino ${ }^{10}$.

O padrão de continuidade de canhotismo e destrimanismo na evolução da espécie humana, bem como a distribuição entre os sexos sugeriram a influência de fatores biológicos e apontaram para uma possível etiologia genética $^{6,11,12}$. Por extensão, ao nível fenotípico, os canhotos quase invariavelmente tendem a ser menos consistentes do que os destros ${ }^{13-15}$, ou seja, não efetuam a maioria das tarefas que requeiram coordenação motora fina e/ou força muscular com a mão esquerda, como os destros efetuam com a direita.

Segundo a terminologia genética, dominância manual seria uma expressão fenotípica do traço, enquanto o padrão de controle neuromotor subjacente, teoricamente seria uma função genotípica ${ }^{6,12}$. No caso de gêmeos, há poucas pesquisas na literatura científica sobre a maioria das características laterais supracitadas, inclusive dominância manual consistente versus dominância manual mista (o uso da mão esquerda na execução de algumas tarefas e a direita em outras).

A causa do fenômeno da dominância manual e as demais preferências laterais que a espécie humana manifesta, ainda suscitam controvérsias entre os cientistas da área, pois, evidenciam questionamentos seculares sobre a correlaçấo entre a genética e o ambiente. Neste contexto, o estudo de gêmeos tem sido uma possibilidade de analisar o efeito da hereditariedade e do ambiente por permitir investigar a contribuição diferencial dos fatores genéticos e ambientais no desenvolvimento de certos traços e comportamentos $^{16,17}$.

Sumariamente, o procedimento básico requer a comparação da razão de concordância entre monozigóticos (MZs), classificado como idêntico ou univitelino e, dizigóticos (DZs), também identificados como fraternos ou bivitelinos. Assim, quaisquer diferenças (discordâncias) entre $M Z$ s decorrem de fatores não genéticos, enquanto a diferença entre DZs deve-se tanto a fatores genéticos quanto ambientais, por conseguinte, à medida que o nível de concordância entre os MZs supera o nível de concordância entre DZs,verifica-se a contribuição genética do atributo sob estudo ${ }^{16,17}$.

Quando o traço pode ser medido em uma escala contínua, seria possível medir a direção e o grau das diferenças e semelhanças, através da ANOVA e o coeficiente de correlação intraclasse $\left(r_{i c}\right)$; quanto maior o valor da $r_{i c}$, maior o nível de concordância entre gêmeos e, portanto, a proporção de variância atribuível a fatores genéticos, enquanto $1-\mathrm{r}_{\mathrm{ic}}$ reflete a proporçẫo atribuível ao ambien$\mathrm{te}^{16,17}$.

Infelizmente, como será demonstrado em uma seção posterior, no caso de dominância manual, os dados de gêmeos tem sido um obstáculo na formação de qualquer teoria genética. Com base nos achados de diversas pesquisas desde a década de 1920, a taxa de discordância (onde um irmão gêmeo é destro e o outro canhoto) tem sido relativamente alta $(\approx 22 \%)$, independentemente de zigosidade $^{18,19}$. Por conseguinte tentativas de conciliar esta tendência com qualquer modelo genético simples tem suscitado controvérsias.

Diante da questão exposta, o objetivo deste artigo foi realizar uma revisão de literatura referente à dominância manual (destro/canhoto) de gêmeos monozigóticos e dizigóticos e as teorias envolvidas na organização motora. 


\section{MÉTODO}

Realizou-se uma revisão de literatura através da seleção de periódicos e livros científicos internacionais e nacionais que abordassem as assimetrias laterais, principalmente a dominância manual em gêmeos, no entanto, priorizou-se as referências internacionais, visto que, na literatura nacional esta área de pesquisa com gêmeos ainda é incipiente. Utilizou-se as bases de dados COMUT/ BIREME, LILACS, Science Direct, Pubmed, Coogprints e Cochrane. Considerou-se como critérios de inclusão, estudos de meta-análise, revisóes de literatura e revisôes sistemáticas desenvolvidos com gêmeos monozigóticos e dizigóticos, sem restrição de faixa etária de ambos os gêneros, com desenvolvimento neuropsicomotor normal. Os critérios de exclusão foram gêmeos teratópagos, presença de diagnósticos (motor, sensorial, cognitivo, comportamental, etc). "Os unitermos empregados nas buscas foram palavras da língua inglesa, tais como: "handedness", "brain asymmetry"," handedness in twins", "twins" "functional laterality", "zygosity" e "laterality" . A data da publicação variou entre o século XX e XXI, ou seja, não houve limitação de datas. Os textos foram analisados e sumariados criticamente.

\section{RESULTADOS}

\section{Assimetrias Laterais entre Gêmeos}

De modo geral, os dados primários refletem dados nominais ou categóricos e não é possível calcular a $\mathrm{r}_{\mathrm{ic}}$ (coeficiente de correlação intraclasse), para testar o nível de concordância, embora seja possível obter estimativas de associação e concordância por meio de uma versão do coeficiente fi $(\Phi)$ adaptado para a análise de traços dicotômicos em gêmeos ${ }^{11}$. Assim, segundo a expectativa de uma teoria de determinação genética, em MZs, o valor de $\Phi$ deve ser alto e, em DZs menor, mas acima de zero. Um coeficiente em torno de zero indica uma distribuição binominal, ou seja, o traço ocorre aleatoriamente e devese a fatores não genéticos.

Para exemplificar as tendências básicas, apresentam-se na Tabela 1 os dados extraídos de 11 estudos publicados entre o ano de 1970 e 2009 . Estes artigos foram escolhidos porque, na maioria deles, a zigosidade foi determinada por métodos biológicos atualizados (ex: exames de sangue) tomando o erro de classificação da zigosidade baixo $(\approx 5 \%)$. Ao combinar estes dados, a fim de simplificar a descrição, foi exposta a mediana das percentagens em cada categoria.

Tabela 1

Dominância manual em gêmeos monozigóticos e dizigóticos: 1970 a 2009

\begin{tabular}{|c|c|c|c|c|c|c|c|}
\hline Estudo & Zigos & $\mathrm{Np}$ & DD & DC & $\mathrm{CC}$ & $\mathbf{N}_{\text {Total }}$ & $\mathbf{N}_{\text {Can }}$ \\
\hline \multirow{2}{*}{20} & $\mathrm{MZ}$ & 187 & $\begin{array}{c}132 \\
(70,6)\end{array}$ & $\begin{array}{c}46 \\
(24,6)\end{array}$ & $\begin{array}{c}09 \\
(4,8) \\
\end{array}$ & 374 & $\begin{array}{c}64 \\
(17,1)\end{array}$ \\
\hline & $\mathrm{DZ}$ & 176 & $\begin{array}{c}115 \\
(65,3)\end{array}$ & $\begin{array}{c}54 \\
(30,7)\end{array}$ & $\begin{array}{c}07 \\
(4,0) \\
\end{array}$ & 352 & $\begin{array}{c}68 \\
(19,3) \\
\end{array}$ \\
\hline \multirow{2}{*}{21} & $\mathrm{MZ}$ & 514 & $\begin{array}{c}380 \\
(74,0) \\
\end{array}$ & $\begin{array}{c}123 \\
(23,9) \\
\end{array}$ & $\begin{array}{c}11 \\
(2,1) \\
\end{array}$ & 1028 & $\begin{array}{c}145 \\
(14,1) \\
\end{array}$ \\
\hline & $\mathrm{DZ}$ & 333 & $\begin{array}{c}261 \\
(78,4)\end{array}$ & $\begin{array}{c}70 \\
(21,0)\end{array}$ & $\begin{array}{c}02 \\
(0,6)\end{array}$ & 666 & $\begin{array}{c}74 \\
(11,1)\end{array}$ \\
\hline \multirow{2}{*}{22} & $\mathrm{MZ}$ & 75 & $\begin{array}{c}53 \\
(70,7) \\
\end{array}$ & $\begin{array}{c}19 \\
(25,3) \\
\end{array}$ & $\begin{array}{c}03 \\
(4,0) \\
\end{array}$ & 150 & $\begin{array}{c}25 \\
(16,7) \\
\end{array}$ \\
\hline & $\mathrm{DZ}$ & 47 & $\begin{array}{c}35 \\
(74,4) \\
\end{array}$ & $\begin{array}{c}09 \\
(19,2) \\
\end{array}$ & $\begin{array}{c}03 \\
(6,4) \\
\end{array}$ & 94 & $\begin{array}{c}15 \\
(16,0) \\
\end{array}$ \\
\hline \multirow{2}{*}{23} & $\mathrm{MZ}$ & 214 & $\begin{array}{c}145 \\
(67,8) \\
\end{array}$ & $\begin{array}{c}45 \\
(21,0) \\
\end{array}$ & $\begin{array}{c}24 \\
(11,2) \\
\end{array}$ & 428 & $\begin{array}{c}93 \\
(21,7) \\
\end{array}$ \\
\hline & $\mathrm{DZ}$ & 214 & $\begin{array}{c}132 \\
(61,7)\end{array}$ & $\begin{array}{c}69 \\
(32,2)\end{array}$ & $\begin{array}{c}13 \\
(6,1)\end{array}$ & 428 & $\begin{array}{c}95 \\
(22,2)\end{array}$ \\
\hline \multirow{2}{*}{24} & $\mathrm{MZ}$ & 96 & $\begin{array}{c}78 \\
(81,3) \\
\end{array}$ & $\begin{array}{c}16 \\
(16,7)\end{array}$ & $\begin{array}{c}02 \\
(2,0) \\
\end{array}$ & 192 & $\begin{array}{c}20 \\
(10,4)\end{array}$ \\
\hline & $\mathrm{DZ}$ & 68 & $\begin{array}{c}44 \\
(64,7)\end{array}$ & $\begin{array}{c}21 \\
(30,9)\end{array}$ & $\begin{array}{c}03 \\
(4,4)\end{array}$ & 136 & $\begin{array}{c}27 \\
(19,9)\end{array}$ \\
\hline
\end{tabular}


Tabela 1

(Continuação)

\begin{tabular}{|c|c|c|c|c|c|c|c|}
\hline Estudo & Zigos & $\mathrm{Np}$ & DD & DC & CC & $\mathbf{N}_{\text {Total }}$ & $\mathbf{N}_{\text {Can }}$ \\
\hline \multirow{2}{*}{25} & $\mathrm{MZ}$ & 197 & $\begin{array}{c}175 \\
(88,8)\end{array}$ & $\begin{array}{c}21 \\
(10,7)\end{array}$ & $\begin{array}{c}01 \\
(0,5)\end{array}$ & 394 & $\begin{array}{c}23 \\
(05,8)\end{array}$ \\
\hline & $\mathrm{DZ}$ & 203 & $\begin{array}{c}171 \\
(84,2)\end{array}$ & $\begin{array}{c}32 \\
(15,8)\end{array}$ & $\begin{array}{c}0 \\
(0,0)\end{array}$ & 406 & $\begin{array}{c}32 \\
(07,9)\end{array}$ \\
\hline \multirow{2}{*}{26} & $\mathrm{MZ}$ & 836 & $\begin{array}{c}655 \\
(78,3)\end{array}$ & $\begin{array}{c}158 \\
(18,9)\end{array}$ & $\begin{array}{c}23 \\
(2,8) \\
\end{array}$ & 1672 & $\begin{array}{c}204 \\
(12,2) \\
\end{array}$ \\
\hline & $\mathrm{DZ}$ & 832 & $\begin{array}{c}626 \\
(75,2)\end{array}$ & $\begin{array}{c}183 \\
(22,0)\end{array}$ & $\begin{array}{c}23 \\
(2,8) \\
\end{array}$ & 1664 & $\begin{array}{c}229 \\
(13,8) \\
\end{array}$ \\
\hline \multirow{2}{*}{27} & $\mathrm{MZ}$ & 352 & $\begin{array}{c}249 \\
(70,8)\end{array}$ & $\begin{array}{c}86 \\
(24,4) \\
\end{array}$ & $\begin{array}{c}17 \\
(4,8) \\
\end{array}$ & 704 & $\begin{array}{c}120 \\
(17,1) \\
\end{array}$ \\
\hline & $\mathrm{DZ}$ & 408 & $\begin{array}{c}276 \\
(67,7)\end{array}$ & $\begin{array}{c}109 \\
(26,7)\end{array}$ & $\begin{array}{c}23 \\
(5,6) \\
\end{array}$ & 816 & $\begin{array}{c}155 \\
(19,0) \\
\end{array}$ \\
\hline \multirow{2}{*}{28} & $\mathrm{MZ}$ & 622 & $\begin{array}{c}475 \\
(76,4) \\
\end{array}$ & $\begin{array}{c}122 \\
(19,6) \\
\end{array}$ & $\begin{array}{c}25 \\
(4,0) \\
\end{array}$ & 1244 & $\begin{array}{c}172 \\
(13,8) \\
\end{array}$ \\
\hline & $\mathrm{DZ}$ & 1041 & $\begin{array}{c}764 \\
(73,4)\end{array}$ & $\begin{array}{c}255 \\
(24,8)\end{array}$ & $\begin{array}{c}22 \\
(2,1)\end{array}$ & 2082 & $\begin{array}{c}299 \\
(14,4) \\
\end{array}$ \\
\hline \multirow{2}{*}{29} & $\mathrm{MZ}$ & 1152 & $\begin{array}{c}956 \\
(82,9) \\
\end{array}$ & $\begin{array}{c}179 \\
(15,6) \\
\end{array}$ & $\begin{array}{c}17 \\
(1,5) \\
\end{array}$ & 2304 & $\begin{array}{c}213 \\
(09,3) \\
\end{array}$ \\
\hline & $\mathrm{DZ}$ & 965 & $\begin{array}{c}826 \\
(85,6)\end{array}$ & $\begin{array}{c}128 \\
(13,3)\end{array}$ & $\begin{array}{c}11 \\
(1,1)\end{array}$ & 1930 & $\begin{array}{c}150 \\
(07,8)\end{array}$ \\
\hline \multirow{2}{*}{30} & $\mathrm{MZ}$ & 2509 & $\begin{array}{c}1923 \\
(76,6) \\
\end{array}$ & $\begin{array}{c}527 \\
(21,0) \\
\end{array}$ & $\begin{array}{c}59 \\
(2,4) \\
\end{array}$ & 5018 & $\begin{array}{c}645 \\
(12,9) \\
\end{array}$ \\
\hline & $\mathrm{DZ}$ & 2752 & $\begin{array}{c}2121 \\
(77,1) \\
\end{array}$ & $\begin{array}{c}567 \\
(20,6) \\
\end{array}$ & $\begin{array}{c}64 \\
(2,3) \\
\end{array}$ & 5504 & $\begin{array}{c}695 \\
(12,6) \\
\end{array}$ \\
\hline \multirow{2}{*}{31} & $\mathrm{MZ}$ & 2509 & $\begin{array}{c}1888 \\
(75,3) \\
\end{array}$ & $\begin{array}{c}532 \\
(21,2) \\
\end{array}$ & $\begin{array}{c}89 \\
(3,5) \\
\end{array}$ & 5018 & $\begin{array}{c}710 \\
(14,2)\end{array}$ \\
\hline & $\mathrm{DZ}$ & 2752 & $\begin{array}{c}2041 \\
(74,2)\end{array}$ & $\begin{array}{c}631 \\
(22,0)\end{array}$ & $\begin{array}{c}80 \\
(2,9)\end{array}$ & 5504 & $\begin{array}{c}791 \\
(14,4)\end{array}$ \\
\hline
\end{tabular}

Estudo $=$ Autores nas referências bibliográficas; Zigos = Zigosidade; $\mathrm{Np}=\mathrm{O}$ Número de Pares $; \mathrm{DD}=$ Ambos Destros; DC $=$ Discordante (um destro/um canhoto); $\mathrm{CC}=$ Ambos Canhotos; NTotal = O Número Total de Gêmeos (i.e. 2 x Np); NCan = O Número Total de Canhotos (i.e. 2 x CC + DD). As Percentagens entre Parênteses.

\section{DISCUSSÃO}

Entre os trabalhos apresentados, fica evidente a variabilidade entre os resultados encontrados. Uma possível explicação baseia-se em alguns fatores identificados: critérios diferentes para classificar destros e canhotos; técnicas diferentes para a mensuração da dominância manual; várias faixas etárias estudadas (de 6 a 90 anos); a distribuição por gênero feminino e masculino.

Ao somar todos os casos através de 11 estudos, no total contabilizou-se 13,793 pares de gêmeos, 6,754 MZs e 7,039 DZs. Com referência a percentagem mediana de concordância e discordância, os MZs DD(destro-destro) igualou-se a 76,4\%, os DC(destro-canhoto) foi de 21,0\% e os CC(canhoto- canhoto) a 2,6\%. Para os DZs DD a percentagem foi $74,4 \%$, os DC resultou em $22 \%$ e os CC a $3,6 \%$.

Ao avaliar estas proporçóes por meio do $\Phi$, obteve-se $\Phi_{\mathrm{MZ}}=0,177$ e $\Phi_{\mathrm{DZ}}=0,089$. Estes coeficientes são inconsistentes, não significantes e por inferência, indicam a ausência relativa de semelhança genética. Além do mais, quando tratados como indivíduos, a prevalência de canhotismo é ligeiramente maior entre os $\mathrm{DZs}$ do que entre os MZs (14,4\% vs. 13,8\%), ou seja, não há uma diferença significativa entre os dois grupos. O grau acentuado de discordância (DC) aparece igualmente entre ambos os tipos de gêmeos. Se considerado estritamente em termos estatísticos, a distribuição binomial significa a presença de variação aleatória em torno de uma alta prevalência 
de destrimanismo. Por outro lado, vale lembrar que esta tendência somente reflete a direção da assimetria manual e por inferência sustenta a hipótese citada acima de que este aspecto de dominância não é hereditário ${ }^{14,32}$.

De acordo com os dados resumidos acima na Tabela 1 , as tendências mais destacadas referem-se à alta taxa de discordância na dominância manual e, em relação aos indivíduos não gêmeos, a alta prevalência de canhotismo. Mediante os dados supracitados, em quase todos os resultados relacionados, a taxa de discordância (DC), embora variável (entre 10 a 32\%), com poucas exceções, não diferiu dos valores esperados de uma distribuição binomial.

No que se refere à suposta prevalência realçada de canhotismo entre gêmeos, os achados não foram tão consistentes. Em uma análise dos dados disponíveis de pesquisas independentes que mensuraram a dominância manual de gêmeos e não gêmeos (i.e. um verdadeiro grupo de controle), identificou-se apenas quatro que usou a mesma medida e o mesmo critério para diferenciar destros de canhotos ${ }^{33}$. Após ter combinado as frequências, a incidência de canhotismo foi ligeiramente maior entre os gêmeos do que os não gêmeos, mas, a diferença não foi significativa. Em contraste, quase 20 anos depois, alguns autores $^{19}$ reuniram os dados de 12 pesquisas compostas de gêmeos e não gêmeos.

Em oito dos 12 estudos, em comparação com os não gêmeos, a taxa de canhotismo foi significativamente elevada em gêmeos e como resultado a meta-análise subsequente dos dados combinados, revelou uma diferença global significativa, em apoio à hipótese de uma prevalência maior de canhotismo em gêmeos. Dos estudos mais recentes, dois encontraram a presença desta tendência ${ }^{34,35}$, enquanto três não registraram divergência alguma $^{30,31,36}$. Convém, no entanto, enfatizar que existem problemas associados com alguns recentes achados.

Apesar do grande tamanho da amostra de gêmeos $^{30,31}$, obtiveram os dados agrupados de diversos levantamentos independentes sobre assuntos diferentes, embora dominância manual (como o escrever e arremessar uma bola) fosse avaliada neste estudo, o grupo de não gêmeos era formado pelos irmãos dos gêmeos, uma amostra apropriada para a análise de correlatos genéticos, mas, para fins de validade externa (generalização para a população geral) precisa-se de um agregado de não gêmeos sem nenhum vínculo consanguíneo.

Por extensão, entre os 12 estudos $^{19}$ houve um ${ }^{25}$ que também usou a dominância manual dos parentes do primeiro e segundo grau nas comparaçóes, em vez de um verdadeiro grupo de controle. Por fim, uma pesquisa realizada em Taiwan ${ }^{36}$, onde historicamente a influência de pressão social contra canhotismo (pelo menos para escrever e comer) é forte, a prevalência de canhotismo foi menos de $2 \%{ }^{37}$.

Além disso, pesquisadores ${ }^{36}$ tabelaram os dados de tal forma que foi impossível deduzir a taxa de canhotismo entre os MZs (a maioria dos gêmeos) e os DZs, que por sua vez foram emparelhados com irmãos não gêmeos. Em outras palavras, sem ter levado em conta o número de canhotos convertidos, não foi possível obter uma estimativa acurada, tanto da prevalência de destrimanismo, quanto do canhotismo nas suas amostras. De modo geral, com a exceção de duas pesquisas ${ }^{35,38}$, nenhuma das pesquisas sobre lateralidade em gêmeos cita a quantidade de canhotos convertidos para escrever com a mão direita.

Outros pesquisadores ${ }^{39}$, como consta na Tabela 1 , aplicaram um inventário composto de 12 itens em gêmeos (75 MZs, $47 \mathrm{DZs}$, idade média por volta dos 17 anos) e náo gêmeos (30 destros e 20 canhotos); a amplitude do escore manual variou entre 12 (fortemente destro) e 70 (fortemente canhoto). As médias deste escore foram apresentadas para os destros e canhotos em cada grupo, mas sem os desvios padróes (impossibilitando uma apreciação do nível de variabilidade). Os escores médios foram quase iguais através dos três grupos de destros $(\mathrm{MZs}, \mathrm{DZ}$ s e não gêmeos) $)^{39}$.

Por outro lado os valores médios entre os canhotos sugeriram que os DZs (com apenas oito indivíduos no subgrupo) foram mais mistos do que os não gêmeos, com os MZs canhotos em uma posição intermediária. Os autores não forneceram nenhuma análise comparativa e, sem os desvios padróes não foi possível a verificação da ocorrência de diferenças significativas entre as médias. Como consta na revisão da tabela, os pesquisadores ${ }^{29}$ analisaram os dados do conjunto de um agregado maior de gêmeos que preencheram um questionário com cinco itens, a fim de avaliar a concordância por causa do grau de consistência manual. 
Em vez de apresentar as médias e os desvios padrôes, os autores tabelaram a frequência dos gêmeos mostrando concordância intrapar em cinco categorias (fortemente destro até fortemente canhoto). Os dados foram cruzados de tal forma que ficou impossível deduzir o número de gêmeos mistos e consistentes, em grande parte porque os mistos foram inseridos nos grupos dos ambidestros. Além do mais, o registro de gêmeos foi constituído de veteranos norte-americanos da segunda guerra mundial, representando uma geração em que o uso da mão esquerda para escrever e comer, foi sujeita, de modo geral, a pressão social intensiva ${ }^{39}$, ou em outras palavras, a estimativa de canhotismo foi muito deflacionada. Este estudo também falhou na falta de apresentação de dados dos grupos dos não gêmeos como grupo controle para a efetuação de comparações válidas.

\section{CONCLUSÃO}

Quando se leva em consideração os dados disponíveis sobre a distribuição das outras assimetrias laterais e traços fenotípicos associados com dominância manual, ficou evidente que, no caso de gêmeos, existe uma carência generalizada de informação, especificamente, dado a importância atribuída à dominância manual, o conhecimento das tendências comparativas entre gêmeos e não gêmeos é escasso e inconsistente.

Não existe um consenso científico sobre as divergências encontradas nos gêmeos em relação à dominância manual, visto que, é impossível, sem dados sobre o grau de assimetria (as médias de um escala contínua), verificar o grau de proficiência manual, a relação entre dominância manual, assimetrias entre parentes do primeiro grau (entre outras variáveis) e chegar a uma conclusão definitiva, quanto ao papel de fatores genéticos ou ambientais.

Sem estimativas mais exatas de distribuição e interação de amostras de gêmeos e não gêmeos, não seria possível avaliar os efeitos sobre a magnitude e direção de assimetrias laterais, bem como a relevância dos resultados para as teorias que procuraram elucidar o padrão enigmático de lateralidade em gêmeos.

É evidente que os resultados supracitados não respondem estes questionamentos, dado que, sem uma descrição compreensível e extensiva das expressôes fenotípi- cas derivadas dos grupos, seria muito difícil avaliar certas hipóteses alternativas referentes às possíveis variaçōes no padrão de especializaçóes neuromotoras em gêmeos.

Diante do panorama apresentado, a dominância manual em gêmeos, trata-se indiscutivelmente de um alvo para futuras pesquisas, pois, a compreensão das manifestaçôes laterais humana esclarecerá muitas questóes sobre o próprio desenvolvimento do indivíduo.

\section{REFERÊNCIAS}

1.Barnsley RH, Rabinovitch MS. Handedness: Ptoficiency versus stated preference. Perceptual and Motor Skills 1970;30:343-62.

http://dx.doi.org/10.2466/pms.1970.30.2.343

2.Dassonville P, Zho X, Urgubil K, Kim S, Ashe J. Functional activation in motor cortex affects the direction and degree of handedness. Proceedings of the National Academy of Sciences, USA 1997;94:14015-8.

http://dx.doi.org/10.1073/pnas.94.25.14015

3.Klöppel S, Vongerichte A, Van Eimeren T, Frackowiak RSJ, Siebner HR. Can left-handedness be switched? Insights from early switch of handwriting. Journal of Neuroscience 2007;27:7847-53.

http://dx.doi.org/10.1523/JNEUROSCI.1299-07.2007

4.Rijntjes M, Dettmers C, Büchel C, Kielbel S, Frackowiak RSJ, Weiller C. A blueprint for movement: Functional and anatomical representations in the human motor system. Journal of Neuroscience 1999;19:8043-8.

5.Singh LN, Takahahi S, Kurihara N, Furuta S, Tamura H. Ckomparison of ipsilateral activation between right- and left-handers: A functional MR study. NeuroReport 1998;9:1861-6.

http://dx.doi.org/10.1097/00001756-199806010-00036

6.Bradshaw JL. Human evolution: A neuropsychological perspective. NY; 1997, p.120-37.

7.Pobiner BL. The use of stone tools to determine handedness in hominids. Current Anthropology 1999;40:90-2.

http://dx.doi.org/10.1086/515807

8.Rugg G, Mullane M. Inferring handedness from lithic evidence. Laterality 2001;6:247-59.

http://dx.doi.org/10.1080/13576500042000188

http://dx.doi.org/10.1080/713754411

9. Toth N. Archaeological evidence for preferential right-handedness in the lower and middle Pleistocene and its possible implications. Journal of Human Evolution 1985;14:607-14.

http://dx.doi.org/10.1016/S0047-2484(85)80087-7

10.Papadatou-Pastou M, Martin M. Sex differences in left-handedness: A meta-Analysis of 144 studies. Psychological Bulletin 2008;134:677-99.

http://dx.doi.org/10.1037/a0012814

11.Corballis, MC. From hand to mouth: The origins of language. Princeton, NJ: Princeton University Press; 2002, p.200-41.

12.McManus IC. Right hand, left hand: The origins of asymmetry in brains, bodies, atoms and cultures. Cambridge, MA: Harvard University Press; 2004, p.380-20.

13.Beaton A. The nature and determinants of handedness. In K. Hugdahl RJ, Davidson. The asymmetrical brain. Cambridge, MA: Bradford; 2003, p.104-55. 
14.Bryden MP, Steenhuis RE. Issues in the assessment of handedness. In: Kitterle F. Cerebral Laterality:Theory and research.Hillsdale NJ: L. Erlbaum; 1991, p.35-52.

15.Schachter SC. The quantification and definition of handedness: Implications for handedness Research. In: Mandal MK, Bulman-Fleming MB, Tiwari G. Side bias: A neuropsychogical perspective; 2000, p.155-74.

16.Bulmer MC. The biology of twinning in man. Oxford: Clarendon Press, 1970, p.150-60.

17.Mittler P. The study of twins. Harmondsworth: Penguin Education; 1971, p.170-9.

18.McManus IC, Bryden MP. The genetics of handedness, cerebral dominance, and lateralization. In: Rapin I, Handbook of neuropsychology. Amsterdam: Elsevier; 1992, p.115-44.

19.Sicotte NL, Woods RP, Mazziotta JC. Handedness in twins: A meta-analysis. Laterality, 1999;4:265-86.

http://dx.doi.org/10.1080/713754339

http://dx.doi.org/10.1080/135765099396980

20.Carter-Saltzman L, Scarr-Salapatek S, Barker WB, Katz S. Left-handedness in twins: Incidence and patterns of performance in an adolescent sample. Behavior Genetics.1976;6:189-203.

http://dx.doi.org/10.1007/BF01067148

21.Loehlin JC, Nichols RC. Heredity, environment and personality: A study of 850 sets of twins. Austin, TX: University of Texas Press; 1976, p.202-50.

22.Springer SP, Searleman A. Laterality in twins: The relationship between handedness and hemispheric asymmetry for speech. Behavior Genetics 1978;8:349-57.

http://dx.doi.org/10.1007/BF01067398

23.Boklage CF. Interactions between opposite-sex dizygotic fetuses and the assumptions of Weinberg difference method epidemiology. American Journal of Human Genetics 1985;37:591-605.

24.Forrai G, Bankovi G. A Hungarian twin study on hand clasping, arm folding and tongue curling. Acta Biologica Hungarica 1983;34:99-106.

25.Tambs K, Magnus P, Berg K. Left-handedness in twin families: Support of an environmental hypothesis. Perceptual and Motor Skills 1987;64:155-70. http://dx.doi.org/10.2466/pms.1987.64.1.155

26.Neale MC. Handedness in a sample of volunteer twins. Behavior Genetics.1988;18:69-79.

http://dx.doi.org/10.1007/BF01067076

27.Derom C, Thiery E, Vlietinck R, Loos R, Derom R. Handedness in twins according to zygosity and chorion type: A preliminary report. Behavior Genetics. 1996;26:407-8.

http://dx.doi.org/10.1007/BF02359484
28. Orlebeke JF, Knol DL, Koopmans JR, Boomsa DI, Bleker OP. Left-handedness in twins: Genes or environment? Córtex.1996;32:479-90.

29. Ross DC, Jaffe J, Collins RL, Page W, Robinette D. Handedness in the NAS/NRC twin study. Laterality 1999;4:257-64.

http://dx.doi.org/10.1080/713754342

http://dx.doi.org/10.1080/135765099396971

30.Medland SE, Wright MJ, Geffen GM, Hay DA, Levy F, Martin NG, et al. Special twin environments, genetic influences and their effects on the handedness of twins and their siblings. Twin Research 2003;6:119-30.

http://dx.doi.org/10.1375/136905203321536245

31.Medland SE, Duffy DL, Wright MJ, Geffen GM, Hay DA, Levy F, et al. Genetic influences on handedness: Data from 25,732 Australian and Dutch twin families. Neuropsychologia 2009;47:330-7.

http://dx.doi.org/10.1016/j.neuropsychologia.2008.09.005

32.Collins RL. On the inheritance of direction and degree of asymmetry. In: S.D. Glick. Cerebral lateralization in nonhuman species. NY: Academic Press.1985; p.41-72.

33.McManus IC.Handedness in twins: A critical review. Neuropsychologia 1980; $18: 347-55$.

http://dx.doi.org/10.1016/0028-3932(80)90130-X

34.Ooki S. Genetic and Environmental influences on the handedness and footedness in Japanese twin children. Twin Research and Human Genetics.2005;8:649-56.

http://dx.doi.org/10.1375/twin.8.1.69

http://dx.doi.org/10.1375/twin.8.4.320

http://dx.doi.org/10.1375/twin.8.6.649

35.Vuoksimaa E, Koskenvuo M, Rose RJ, Kaprio J. Origins of handedness: A nationwide study of 30,161 adults. Neuropsychologia 2009; 47:1294-301. http://dx.doi.org/10.1016/j.neuropsychologia.2009.01.007

36.Su CH, Kuo PH, Lin CCH, Chen WJ. A school-based twin study of handedness among adolescents in Taiwan. Behavior Genetics. 2005;35:723-33. http://dx.doi.org/10.1007/s10519-005-6189-1

37.Teng EH, Lee PH, Yang KS, Chang PC. Lateral preferences for hand, foot, and eye, and their lack of association with scholastic achievement in 4143 Chinese. Neuropsychologia.1979;17:41-8. http://dx.doi.org/10.1016/0028-3932(79)90020-4

38.Shimizu A, Endo M. Comparison of patterns of handedness between twins and singletons in Japan. Cortex. 1983;19:345-52.

39.Springer SP, Searleman A. Left-handedness in twins: Implications for the mechanisms underlying cerebral asymmetry of function. In: Herron J. Neuropsychology of left-handedness. NY: Academic Press; 1980, p.139-58. 